\title{
Estudo Experimental do Momento de Inércia de uma Placa
}

(Experimental study of the moment of inertia of a plate)

\author{
Carlos A. F. Pintão e Moacir P. de Souza Filho \\ Departamento de Física, Unesp - Bauru \\ C.P.473, 17033-360, Bauru, SP, Brasil \\ e-mail: fonzar@fc.unesp.br
}

Recebido em 20 de fevereiro, 2002. Revisado em 22 de outubro, 2002. Aceito em 25 de outubro, 2002.

\begin{abstract}
Neste trabalho, mostra-se um caminho diferente para se estudar o momento de inércia de um corpo em rotação. Descreve-se um experimento que permite estabelecer como a inércia de uma placa depende de sua massa e geometria, em que se determina parâmetros como os expoentes e a constante da tradicional equação do momento de inércia de uma placa, a partir de medidas de corrente elétrica. Para isso escolhe-se quatro placas de massa diferentes, mantendo-se em duas delas as mesmas dimensões, enquanto que nas outras duas uma das dimensões é o dobro da outra. Os resultados obtidos reforçam a idéia de que o sistema e o procedimento de medida utilizado podem ser uma alternativa em realizar esta prática nos laboratórios de ensino.
\end{abstract}

In this work, a different way to study the moment of inertia of a body in rotation is shown. It is described an experiment that allows to establish how the inertia of a plate depends on its mass and geometry, where parameters like the exponents and the constant of the traditional equation of the moment of inertia of a plate are determined, starting from the measurements of the electric current. For this purpose it was chosen four plates of different masses, which two of them have the same dimensions, while for the other pair of plates one has the side twice larger than the other one. The obtained results reinforce the idea that such a system and the proposed procedure can be an alternative in accomplishing this experiment in teaching laboratories.

\section{Introdução}

Em um artigo recente, Pintão et al. [1] adotaram um procedimento experimental diferente dos tradicionais como aqueles de Goldemberg [2] e Tyler [3] para determinar o momento de inércia de um disco. Utilizaram um método originalmente proposto por Fleming e Clinton [4] e Bennet [5] para a medir capacitância e determinaram diretamente, a partir de uma leitura de corrente elétrica, a velocidade angular do disco após o corpo suspenso descer de uma determinada altura $h$. Conhecendo-se a velocidade, obtém-se a aceleração angular. Para que isto fosse possível, construíram um circuito $R C_{j}$, com uma chave que é acionada através de um sensor opto-eletrônico (emissor e receptor infravermelho) que emite luz sobre a superfície de um CD fixo ao eixo de rotação e dividido em 24 setores circulares igualmente espaçados (12 superfícies espelhadas e 12 opacas, alternadas entre si). Através de uma das duas posições possíveis desta chave, o capacitor $C_{j}$ irá se carregar ou descarregar. Durante este processo, a freqüência de comutação desta chave, $f_{j}$, pode ser expressa pela relação, $f_{j}=i_{j} / C_{j} V$ em que $i_{j}$ é a corrente medida no medidor analógico DC acoplado ao circuito, e $V$ é a voltagem aplicada por uma fonte DC no capacitor $C_{j}$. No caso deste CD com 24 divisões, a freqüência de rotação do eixo é $1 / 12$ daquela da comutação $f_{j}$, portanto, a velocidade angular associada ao eixo é $w_{j}=\pi f_{j} / 6$. Lembrando que a aceleração, $\alpha_{j}$, pode ser determinada da equação de Torricelli para um movimento circular uniformemente acelerado, como $w_{j}^{2}=2 \alpha_{j} \Delta \theta$, e do fato que a altura $h$ é dada por $h=r \Delta \theta$, então se consegue expressar $\alpha_{j}$ como uma função da corrente ao quadrado. Outros detalhes do circuito utilizado podem ser encontrados na Ref. [1]. Com este procedimento, eliminaram a necessidade de se medir o tempo. O experimento é então repetido, variando-se a massa do corpo suspenso. O momento de inércia, por sua vez, é determinado a partir da inclinação da reta obtida, do gráfico do torque (que é conhecido) versus a aceleração angular. A vantagem do método é que não é preciso se preocupar com o atrito, uma vez que a inclinação da reta não depende 
do torque associado às forças de atrito, além de se poder determiná-lo facilmente. Como avaliação do sistema de medida, Pintão et al. [1] determinaram o momento de inércia de um disco e anel e compararam esses resultados aos valores descritos na literatura [6], verificando um desvio percentual inferior a 1\%. Após esta primeira avaliação experimental, Pintão et al. [7] realizaram um experimento dando um novo enfoque ao sistema de medida e conseguiram estabelecer a dependência do momento de inércia de um corpo (partícula) em relação à sua massa e distância ao eixo de rotação. Os resultados verificados nestes trabalhos $[1,7]$ foram bastante importantes na realização destas novas medidas. Como uma extensão dos trabalhos anteriores, apresenta-se agora uma nova alternativa experimental para determinar os expoentes e a constante da tradicional equação do momento de inércia de uma placa.

\section{II \\ Medida do momento inércia}

De uma maneira geral, na medida do momento de inércia de uma placa, usou-se a montagem esquematizada na Fig.1, onde uma das placas $j$ está fixa ao eixo do sistema rotacional. Inicialmente, adotou-se o mesmo procedimento utilizado por Pintão et al. [1] para determinar a inércia do eixo mais os acessórios (CD e parafuso de fixação). Sabe-se que o torque resultante $\tau$, responsável pelo movimento de rotação do eixo é expresso como

$$
\tau=\tau_{T}-\tau_{\mathrm{ATR}}=I_{\mathrm{EIXO}} \alpha_{0},
$$

em que $\tau_{T}$ e $\tau_{\mathrm{ATR}}$ são, respectivamente, os torques associados à tração $T$ aplicada pelo fio e às forças de

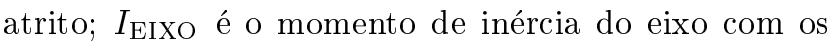
acessórios e $\alpha_{0}$ sua aceleração angular. Com base na Ref. [1] pode-se expressar a aceleração angular de um corpo rígido qualquer em rotação como

$$
\alpha_{j}=k_{j}\left(i_{j}\right)^{2}
$$

em que os sub-índices $j$ significam: $j=0$, a rotação do eixo mais acessórios e, $j=1,2,3$ ou 4 , a rotação do mesmo eixo mais as placas $1,2,3$ ou 4, respectivamente; $k_{j}$ é uma constante conhecida $\left(k_{j}=r \pi^{2} / 72 h\left(C_{j} V\right)^{2}\right)$, uma vez que $r, h, C_{j}$ e $V$ são grandezas previamente determinadas. A força resultante sobre a massa de tração $m$, enquanto está caindo, é $m g-T=m a=m \alpha_{j} r$, de modo que $T=m\left(g-\alpha_{j} r\right)$. Como $\tau_{T}=T r$, resulta $\tau_{T}=m\left(g-\alpha_{j} r\right) r$, que, usando a Eq. (2), pode também ser expresso em termos de corrente como

$$
\tau_{T}=m g r-m k_{j}(r)^{2}\left(i_{j}\right)^{2} .
$$

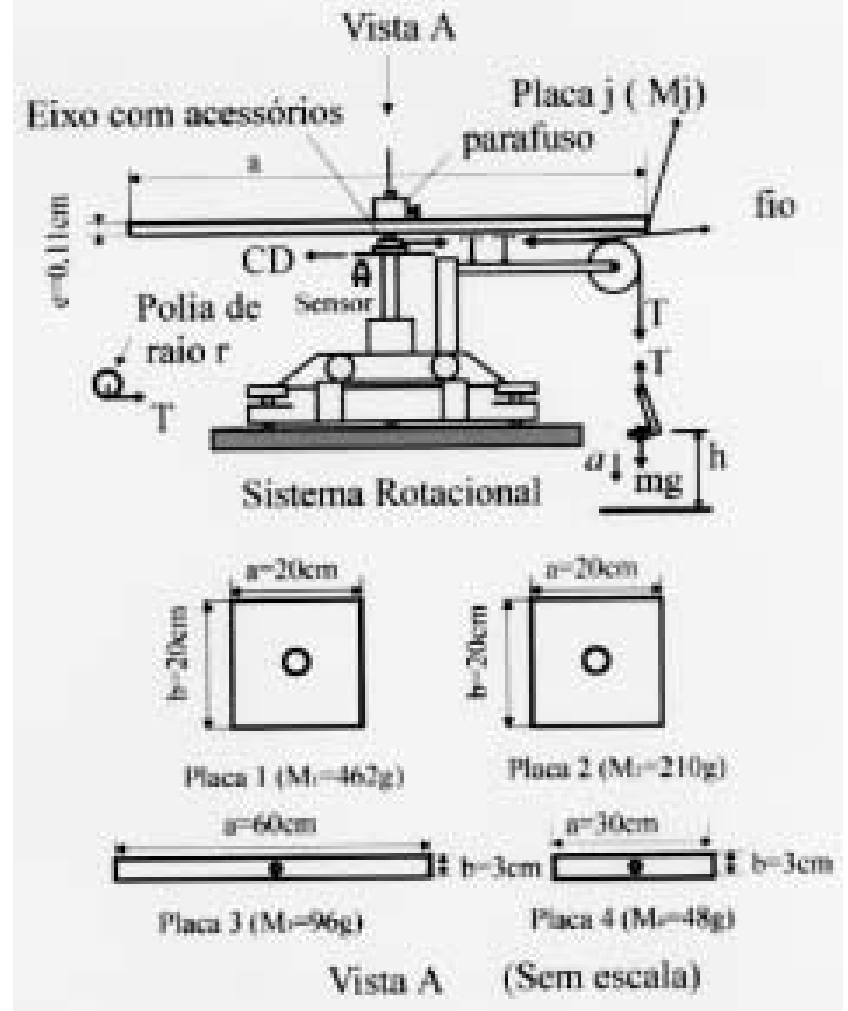

Figura 1. Sistema rotacional com acessórios e, placas de diferentes massas $M_{j}$ (detalhes na vista A).

Assim, conhecendo-se as constantes que comparecem nas Eqs. (2) e (3), pode-se determinar tanto a aceleração angular como o torque, a partir da leitura da corrente. Variando a massa do corpo suspenso e mantendo constante as demais grandezas, pode-se obter um conjunto de valores de $\tau_{T}$ versus $\alpha_{j}$. Pela Eq. (1), a relação entre essas grandezas é linear e a inclinação da reta fornece o momento de inércia do eixo, $I_{\text {EIXo. A }}$ partir do instante em que a massa de tração $m$ deixa de atuar no movimento do eixo, a Eq. (1) pode ser expressa como

$$
\tau=-\tau_{\mathrm{ATR}}=I_{\mathrm{EIXO}} \alpha_{0} .
$$

Se adicionarmos uma placa de massa $M_{j}$, cujo momento de inércia é $I_{\mathrm{PLACAj}}$, que gira solidária ao eixo (ver Fig. 1) e repetir o procedimento anterior, no instante que a mesma massa deixar de atuar no movimento desse corpo, a Eq. (1) pode ser modificada para

$$
\tau=-\tau_{\mathrm{ATR}}=I_{(\mathrm{PLACAj}+\mathrm{EIXO})} \alpha_{j},
$$

em que $I_{\text {(PLACAj+EIXO) }}$ é o momento de inércia do conjunto placa e eixo.

Considerando que a massa presa na extremidade do fio é a mesma para as duas situações descritas pelas Eqs. (4) e (5), da igualdade $I_{\mathrm{PLACAj}}=I_{(\mathrm{PLACAj}+\mathrm{EIXO})^{-}}$

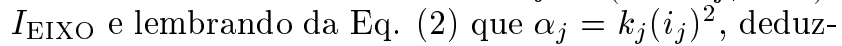
se uma expressão para o momento de inércia da placa $j$, válida para o sistema de medida utilizado: 


$$
I_{\mathrm{PLACAj}}=I_{\mathrm{EIXO}} A_{j}
$$

em que $A_{j}=\left[k_{0} / k_{j}\left(i_{0} / i_{j}\right)^{2}-1\right]$.

As grandezas $i_{0}$ e $i_{j}$ são as correntes medidas nas duas situações descritas pelas Eqs. (4) e (5). Os valores de $k_{0}$ e $k_{j}$ referem-se às constantes fixadas para realizar as medidas, sem e com a adição da placa $j$ no sistema rotacional, respectivamente. Através dos valores experimentais substituídos na Eq. (6), e conhecendo-se previamente a inércia de rotação do sistema (eixo mais acessórios), é possível avaliar o momento de inércia do corpo que está girando. Como proposta deste artigo, realizou-se uma prática que permite estabelecer como o momento de inércia de uma placa depende de sua massa $M_{j}$, de suas dimensões (a e b) e quanto vale a constante de proporcionalidade (B) da tradicional equação para o cálculo da inércia [6]

$$
I_{\mathrm{PLACAj}}=B\left(M_{j}\right)^{X}\left[(a)^{Y}+(b)^{Y}\right]
$$

onde $X, Y$ e $B$ são os parâmetros a serem determinados.

Estrategicamente, foram utilizadas 4 placas (cujas dimensões estão definidas na vista A da Fig. 1), e estabelecemos as seguintes equações para cálculo dos expoentes $X$ e $Y$ da Eq.(7):

$$
X=\left[1 / \log \left(M_{2} / M_{1}\right)\right] \log \left(I_{\mathrm{PLACA}} 2 / I_{\mathrm{PLACA}} 1\right) \mathrm{e}
$$

$$
Y=(1 / \log 2)\left[\log \left(I_{\mathrm{PLACA}} 3 / I_{\mathrm{PLACA}} 4\right)+X \log \left(M_{4} / M_{3}\right)\right]
$$

É importante mencionar que as Eqs. (8) e (9) surgem da (7), levando-se em consideração a escolha adequada de dois pares de placas, sendo todas de massas diferentes porém com um par de placas de mesmas dimensões e o outro par com uma placa possuindo um dos lados duas vezes maior do que um lado correspondente a outra.

O valor da constante $B$ pode ser obtido da Eq. (7), usando o resultado da Eq. (6) (momento de inércia da placa) para quaisquer uma das placas, a massa $\left(M_{j}\right)$, as dimensões $(a$ e $b)$ e os valores dos expoentes $X$ e $Y$ calculados das Eqs. (8) e (9).

Utilizou-se cinco massas de tração diferentes para aplicar o torque associado à força $T$. Isto de fato é necessário para se obter o momento de inércia do eixo ( $\left.I_{\text {EIXO }}\right)$. No entanto, conhecendo-se $I_{\text {EIXO }}$, com o sistema de medida utilizado, não é preciso usar todas essas massas para determinar os parâmetros da Eq. (7). Este último procedimento pode ser vantajoso em casos em que se tenha pouco tempo para realizar uma prática em laboratório. Em nossas medidas, utilizou-se sempre as cinco massas para poder aplicar a teoria estatística de pequenas amostras [8] na avaliação dos erros associados aos parâmetros $X, Y$ e $B$.

\section{Resultados}

\section{III.1 Momento de inércia do eixo com acessórios $\left(I_{\text {EIXO }}\right)$}

Na Fig. 2, utilizando o método dos mínimos quadrados, representou-se a reta que melhor se ajusta aos valores experimentais do torque em função da aceleração angular. O valor encontrado para a inclinação desta reta representa o momento de inércia do eixo e seu valor é $I_{\text {EIXO }}=(0,0055 \pm 0,0001) 10^{-2} \mathrm{kgm}^{2}$, enquanto o coeficiente linear, que corresponde ao valor do torque de atrito, é $\tau_{\text {ATR }}=(0,052 \pm 0,008) 10^{-2} \mathrm{Nm}$.

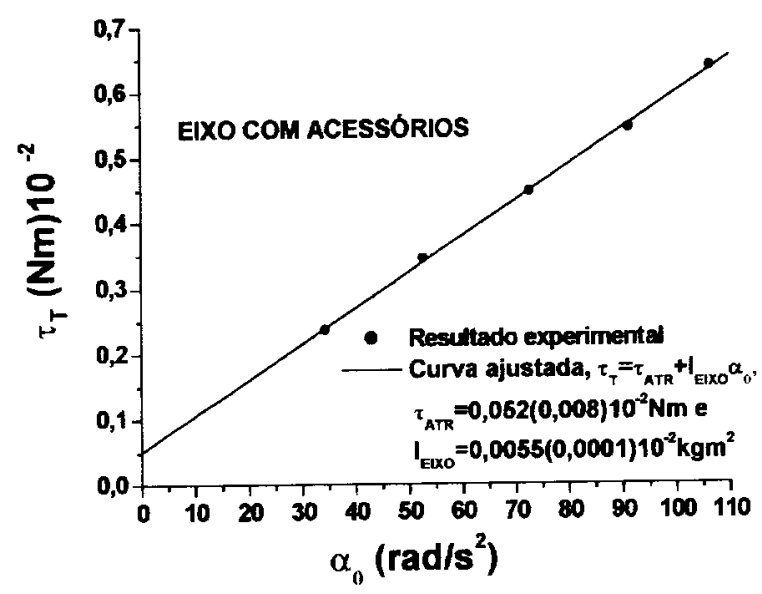

Figura 2. Curvas e pontos experimentais do torque $\tau_{T}$ em função da aceleração angular $\alpha$. A inclinação da reta ajustada pelo método dos mínimos quadrados representa o momento de inércia do eixo com acessórios $I_{\text {EIXO }}$ e, o coeficiente linear, o torque de atrito $\tau_{\mathrm{ATR}}$.

\section{III.2 Estudo do momento de inércia da placa}

III.2.1 Dependência com a massa da placa $\left(M_{j}\right)$ ou cálculo do expoente $X$

Utilizando-se duas placas de mesma dimensões $(a=$ $b=(20,0 \pm 0,1) \mathrm{cm})$ com massas de valores $M_{1}=$ $(461,74 \pm 0,01) \mathrm{g}$ e $M_{2}=(210,22 \pm 0,01) \mathrm{g}$, sendo uma de aço (placa 1) e outra de alumínio (placa 2), conseguiuse manter a mesma geometria durante as medidas. $\mathrm{Na}$ Tabela 1, encontram-se os valores das massas de tração $m$, intensidade das correntes elétricas $i_{0}, i_{1}$ e $i_{2}$, e os parâmetros $A_{1}, A_{2} \mathrm{e} X$. No cálculo dos parâmetros $A_{1}$ e $A_{2}$, estão presentes relações como $k_{0} / k_{1}, k_{0} / k_{2}, i_{0} / i_{1}$ e $i_{0} / i_{2}$. Sabemos que $k_{j}=r \pi^{2} / 72 h\left(C_{j} V\right)^{2}$ é uma constante característica de nosso sistema [1], e que, antes de efetuar qualquer medida de corrente elétrica, devese fixar adequadamente todas estas grandezas. Usouse para todas as medidas deste artigo, uma polia de raio $r=(1,25 \pm 0,01 \mathrm{~cm}$, uma fonte com voltagem $V$ de $(5,1 \pm 0,1)$ volts e uma altura de queda $h$ de $(0,950 \pm 0,005) \mathrm{m}$. Nas medidas de corrente elétrica, em 
que se considerou só o movimento do eixo sem a placa $j$, fixou-se uma capacitância $C_{0}=(32,72 \pm 0,01) \mathrm{nF}$, um pouco menor que $C_{j}=(95,50 \pm 0,01) \mathrm{nF}$ ( $j$ de 1 a 4 , correspondendo a cada uma das placas). Com este procedimento, conseguiu-se evitar que o ponteiro do medidor ultrapassasse o fundo de escala $(50 \mu \mathrm{A}$ e resistência interna $1 \mathrm{k} \Omega$ ). As constantes de tempo, embora sejam levemente diferentes no circuito $R C_{j}$ deste sistema de medida [1], não interferem na leitura da corrente elétrica, pois há tempo suficiente para que o capacitor carregue ou descarregue totalmente. Os valores de corrente e voltagem foram determinados com um mesmo medidor para evitar erro sistemático na sua leitura. Finalmente, os valores $X$ da última coluna, da Tabela 1 , foram calculados através da Eq. (8). O valor encontrado para $X$, com um nível de confiança ${ }^{1}$ de $95 \%$ é $0,96 \pm 0,05$. Pequenas variações na geometria das placas (paralelismo das faces, angulação dos cantos e furos centrados) justificam o fato de $X$ ser levemente diferente de 1 , valor previsto de literatura [6].

Tabela 1 - Valores utilizados na Eq. (8) para determinar $X$, sendo $M_{2} / M_{1}=0,455 ; k_{0} / k_{1}=k_{0} / k_{2}=9,25 \mathrm{e}$ $I_{\mathrm{EIXO}}=(0,0055 \pm 0,0001) 10^{-2} \mathrm{kgm}^{2}$.

\begin{tabular}{ccccccc}
\hline$m(g)$ & $i_{0}(\mu \mathrm{A})$ & $i_{1}(\mu \mathrm{A})$ & $i_{2}(\mu \mathrm{A})$ & $A_{1}$ & $A_{2}$ & $X$ \\
\hline 20,29 & 23,0 & 10,0 & 14,0 & 47,9 & 24,0 & 0,881 \\
\hline 30,29 & 28,5 & 12,0 & 17,5 & 51,2 & 23,5 & 0,987 \\
\hline 40,29 & 33,5 & 14,0 & 20,5 & 51,9 & 23,7 & 0,998 \\
\hline 50,29 & 37,5 & 16,0 & 23,0 & 49,8 & 23,6 & 0,950 \\
\hline 60,29 & 40,5 & 17,5 & 25,5 & 48,5 & 22,3 & 0,987 \\
\hline
\end{tabular}

III.2.2 Dependência com as dimensões a e b da placa ou cálculo do expoente $Y$

Com os valores das massas das placas 3 e 4 , isto é $M_{3}=(96,29 \pm 0,01) \mathrm{g}$ e $M_{4}=(47,64 \pm 0,01) \mathrm{g}$, sendo a dimensão $a=(60,0 \pm 0,1) \mathrm{cm}$ da placa 3 , o dobro da respectiva dimensão da placa 4 (ver vista A da Fig. 1), foi possível deduzir a Eq. (9), que permitiu calcular $Y$. Usou-se, para o cálculo de $Y$, o valor de $\mathrm{X}$ encontrado no item 3.2.1, ou seja $0,96 \pm 0,05$. Na Tabela 2, encontram-se os valores das massas $m$ de tração, intensidade das correntes $i_{0}, i_{3}$ e $i_{4}$ e os parâmetros $A_{3}$, $A_{4}$ e $Y$. Os valores de $m$ e $i_{0}$ utilizados para a determinação do expoente $X$ (tabela 1 ) foram os mesmos para este caso (determinação do expoente $Y$ ), sendo necessário este procedimento para a validade do método. Os parâmetros $A_{3}$ e $A_{4}$ foram calculados levando-se em consideração as relações $k_{0} / k_{3}, k_{0} / k_{4}, i_{0} / i_{3}$ e $i_{0} / i_{4}$. Avaliou-se o erro associado a $Y$, com um nível de confiança de $95 \%$, e encontrou-se que o valor de $Y$ é $2,1 \pm$ 0,1 .
Tabela 2 - Valores utilizados na Eq. (9) para determinar $Y$, sendo $M_{4} / M_{3}=0,495 ; k_{0} / k_{3}=k_{0} / k_{4}=9,25 ; X=$ $0,96 \pm 0,05$ e $I_{\text {EIXO }}=(0,0055 \pm 0,0001) 10^{-2} \mathrm{kgm}^{2}$.

\begin{tabular}{ccccccc}
\hline $\mathrm{m}(\mathrm{g})$ & $i_{0}(\mu \mathrm{A})$ & $i_{3}(\mu \mathrm{A})$ & $i_{4}(\mu \mathrm{A})$ & $A_{3}$ & $A_{4}$ & $Y$ \\
\hline 20,29 & 23,0 & 9,5 & 26,0 & 53,2 & 6,24 & 2,1 \\
\hline 30,29 & 28,5 & 12,0 & 32,0 & 51,2 & 6,34 & 2,04 \\
\hline 40,29 & 33,5 & 14,0 & 37,5 & 51,9 & 6,38 & 2,05 \\
\hline 50,29 & 37,5 & 15,5 & 42,0 & 53,1 & 6,37 & 2,09 \\
\hline 60,29 & 40,5 & 17,0 & 46,0 & 51,5 & 6,17 & 2,09 \\
\hline
\end{tabular}

\section{III.2.3 Cálculo do fator B para a placa 1}

Para determinar B relativo a placa 1 (aço), calculouse o valor médio de $A_{1}$ com base na Tabela 1 , e aplicouse a teoria estatística de pequenas amostras, com um nível de confiança de 95\%, para avaliar o seu erro. Encontrou-se que $A_{1}$ é $50 \pm 2$. Em seguida, com a inclinação da reta, da Fig. 2, determinou-se momento de inércia do eixo, ou seja: $I_{\text {EIXO }}=(0,0055 \pm 0,0001) 10^{-2}$ $\mathrm{kgm}^{2}$. Substituiu-se este valor e o anterior $\left(A_{1}=\right.$ $50 \pm 2$ ) na Eq. (6), e determinou-se o momento de inércia da placa 1 , isto é: $I_{\mathrm{PLACA} 1}=(0,28 \pm 0,01) 10^{-2}$ $\mathrm{kgm}^{2}$. Utilizando todas as grandezas relativas à placa 1 , massa $\left(M_{1}=(461,74 \pm 0,01) \mathrm{g}\right)$, dimensões $(a=b=(20,0 \pm 0,1) \mathrm{cm})$, expoentes $(X=0,96 \pm 0,05 \mathrm{e}$ $Y=2,1 \pm 0,1)$ e o respectivo valor de momento de inércia $\left(I_{\mathrm{PLACA} 1}=(0,28 \pm 0,05) 10^{-2} \mathrm{kgm}^{2}\right)$. Então, finalmente, substituindo-as na Eq. (7), encontrou-se que a constante B é $0,083 \pm 0,003$. Este valor concorda bem com a previsão teórica [6], ou seja 1/12.

\section{Conclusão}

Dos resultados encontrados nos itens III.2.1, III.2.2 e III.2.3 pode-se concluir que:

a) O momento de inércia de uma placa de massa $M_{j}$ depende diretamente de sua massa elevada ao expoente $0,96 \pm 0,05$

b) $\mathrm{O}$ momento de inércia da placa de massa $M_{j}$ depende diretamente das suas dimensões a e b, mais explicitamente da forma $a^{Y}+b^{Y}$, sendo que o valor experimental determinado para $Y$ é $2,1 \pm 0,1$;

c) A constante B, determinada experimentalmente, é $0,083 \pm 0,003$;

d) Com base em nossos resultados experimentais, a equação para cálculo do momento de inércia de uma placa (Eq. 7), pode ser expressa como

$$
I=0,083 M^{0,96}\left[a^{2,1}+b^{2,1}\right] .
$$

${ }^{1}$ Da teoria de pequenas amostras em Estatística, o parâmetro $X$ é expresso como: $X=X_{M} \pm 2,13(S / \sqrt{ } N-1)$, onde $X_{M}$ é a média dos cinco valores $X$ da Tabela 1, o número 2,13 têm origem na distribuição de "Student" (ver apêndice III da ref. 8), considerando que existe $95 \%$ de probabilidade de $X$ pertencer ao intervalo estimado; S é o desvio quadratico médio, isto é $S=\sqrt{ }\left(X_{M}-X_{i}\right)^{2} / N$, com $N=5$ e $X_{i}$ sendo os valores de $X$ no intervalo de 1 a $N$. 
Portanto, como conclusão geral, o sistema desenvolvido permite estudar de uma forma simples como o momento de inércia de um objeto depende de sua massa e geometria, e em alguns casos, como este da placa, estabelecer uma equação para o seu cálculo. Logo, com este trabalho experimental, encontrou-se um caminho diferente para estudar o momento de inércia de um corpo em rotação. Os valores encontrados reforçam a idéia de que este sistema de medida pode ser uma ferramenta útil no auxílio de novas práticas dentro dos laboratórios didáticos, principalmente no que se refere ao estudo da dinâmica de rotação.

\section{Agradecimento}

Gostaríamos de agradecer à FUNDUNESP pelo apoio financeiro e a todos os colegas do Departamento de Física pelo incentivo, comentários e sugestões construtivas, dando sempre um voto de confiança e encorajando-nos durante o andamento de nossos trabalhos.

\section{Referências}

[1] PINTÃO, C. A. F, SOUZA FILHO, M. P de., GRANDINI, C. R., HESSEL, R. Medida do momento de inércia de um disco. Rev. Bras. Ens. Fís. 23, 48 (2001).

[2] GOLDEMBERG, J. Física Geral e Experimental. $2^{a}$ ed., São Paulo: Companhia editora Nacional, 1970, p. 481-483, v.1

[3] TYLER, F. A Laboratory Manual of Physics. $4^{a}$ ed., London: Edward Arnold, 1974, p. 22-24.

[4] Fleming, J. A., ClintON, W.C. On the Measurement of Small Capacities and Inductances. Phil. Mag. S. 6, v.5, n.29, p. 493-511, 1903.

[5] BENNET, G. A. G. Electricity and Modern Physics. $2^{a}$ ed., London: Edward Arnold, 1974, p.167-168.

[6] HALLIDAY, D., RESNICK, R. E WALKER, J. Fundamentos de Física: Mecânica. $4^{a}$ ed., Rio de Janeiro: Livros Técnicos e Científicos, 1998, p.249.

[7] PINTÃO, C. A. F, SOUZA FILHO, M. P. de, XAVIER, J. A. Estudo experimental do momento de inércia de uma partícula. SBPN - Scientific Journal. V.5, n.1, p.192-193, 2001.

[8] SPIEGEL, M. R. Estatística. $4^{a}$ ed., Rio de Janeiro: Editôra McGraw-Hill do Brasil, Ltda, 1972, p.312. 